\title{
The hygiene hypothesis revisited
}

\author{
Alexander P. Malyshkin \\ Correspondence: malishkin_54@mail.ru

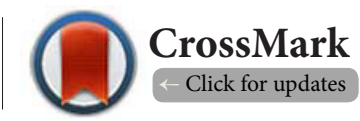

Orenburg State Medical University, 460000 Orenburg, Russia.

\begin{abstract}
To date, a large body of data supports the hygiene hypothesis. However, the explanation of the relationship of autoimmune and allergic diseases with a decrease in microbial load still seems insufficient. Here, an attempt is made at interpreting the available data in terms of a new perspective on infection and immunity.
\end{abstract}

Keywords: Immunology, hygiene hypothesis, mechanisms

\section{Correspondence}

Much evidence for the hygiene hypothesis has been accumulated. The necessity of interaction with ambient microorganisms is interpreted as the necessity of their contact with the immune system, whose insufficient stimulation leads to changes in the ratio between the Th1 and Th2 cell populations, giving rise to autoimmune and allergic disorders. This explanation, however, seems insufficient. There are grounds to believe that the necessity of interaction with microorganisms stems from the usefulness of microbial activities per se rather than immune stimulation [1]. For example, the normal intestinal microflora, which is the most necessary microbial community of the human body, synthesizes vitamins, contributes to food digestion, etc. without involvement of the immune system. The immune system, in this case, should exhibit tolerance to these essential microorganisms rather than be stimulated by them. Normally, it is these microorganisms that should trigger the genetically programmed tuning of the immune system making it tolerant to the essential microflora. Conversely, the absence of the microorganisms should disturb this tuning, which may alter not only the Th1-to-Th2 ratio, but also other parameters of the immune system. However, the pathology that may occur in this case is likely to result not so much from these disturbances as from the absence of the microorganisms whose activities are essential for the host. It is hardly possible yet to explain the exact mechanisms of allergic and autoimmune processes caused by a decrease in microbial load; however, these should result from disturbance of the primary function of the immune system related to normal contacts of immune cells and receptors with self cells and tissues [2]. Every pathological process is a derivative of a normal one and retains some of its components. For example, cardiac arrhythmia implies the existence of the normal rhythm, and respiratory malfunction implies that there is normal respiration. Likewise, the existence of immune pathologies such as allergic and autoimmune diseases suggests the existence of normal interactions of the immune system with self tissues, which are assumed to be part of the primary function of the immune system [2]. Since a consequence of the primary function is that immune cells interact with the determinants of foreign antigens that are similar with those of self antigens [2], interaction with any foreign antigen may be transformed into an autoimmune or allergic process under changed conditions, although the specific mechanisms of this transformation require further study. In this connection, it is reasonable to assume that some ambient microorganisms are involved in the normal interaction of the immune system with self cells and tissues; hence, this interaction may be disturbed when the microbial load is decreased. The development of disease prevention and treatment strategies in the context of the hygiene hypothesis requires identification of the ambient microorganisms that are essential for normal human physiological functions. Given the wide diversity of microorganisms, this is a difficult task; however, the search can be narrowed down by considering that the necessary microorganisms can only be found among infective ones [1].

\section{Competing interests}

The author declares that he has no competing interests.

\section{Acknowledgment}

I am grateful to Professor A. I. Smolyagin and Mr. V. L. Ushakov for the help in the preparation of this paper.

\section{Publication history}

EIC: Dimitrios P. Bogdanos, King's College London School of Medicine, UK.

Received: 05-Aug-2015 Accepted: 01-Sep-2015

Published: 08-Aep-2015

(c) 2015 Alexander P. Malyshkin; licensee Herbert Publications Ltd. This is an Open Access article distributed under the terms of Creative Commons Attribution License (http://creativecommons.org/licenses/by/3.0). This permits unrestricted use, distribution, and reproduction in any medium, provided the original work is properly cited. 
Alexander P. Malyshkin, Immunology Innovation 2015,

http://www.hoajonline.com/journals/pdf/2053-213X-3-1.pdf

doi: 10.7243/2053-213X-3-1

\section{References}

1. Malyshkin AP. Chronic infections: Causes and possible approach to treatment. Res J Infect Dis. 2014; 2:3. | Article

2. Malyshkin AP. Adaptive immunity: The concept of linked functions. Immunollnnov. 2013; 1:1. | Article

\section{Citation:}

Malyshkin AP. The hygiene hypothesis revisited.

Immunol Innov. 2015; 3:1.

http://dx.doi.org/10.7243/2053-213X-3-1 